\title{
Polyelectrolyte Coating of Ferumoxytol Differentially Impacts on Labeling of Inflammatory and Steady-State Dendritic Cell Subtypes
}

\author{
Nehar Celikkin ${ }^{1,2}$, John E. Wong ${ }^{3}$, Martin Zenke ${ }^{1,2}$, and Thomas Hieronymus ${ }^{1,2 *}$ \\ 1 Institute for Biomedical Engineering, Department of Cell Biology, RWTH Aachen University Hospital, \\ Pauwelsstrasse 30, 52074 Aachen, Germany. \\ 2 Helmholtz Institute for Biomedical Engineering, RWTH Aachen University, Pauwelsstrasse 20, 52074 \\ Aachen, Germany. \\ ${ }^{3}$ Institute of Physical Chemistry, RWTH Aachen University, Landoltweg 2, 52056 Aachen, Germany \\ * Corresponding author: \\ E-mail: thomas.hieronymus@rwth-aachen.de \\ Phone: + 492418085249
}

Running title: Labeling of Different DC Subtypes by PE-coated Ferumoxytol

Keywords: Nanoparticles, ferumoxytol, polyelectrolytes, dendritic cells, tracking

\begin{abstract}
Engineered magnetic nanoparticles (MNPs) are emerging as advanced tools for medical applications. The coating of MNPs using polyelectrolytes (PEs) is a versatile means to tailor MNP properties and is used to optimize MNP functionality. Dendritic cells (DCs) are critical regulators of adaptive immune responses. Functional distinct DC subsets exist either under steady-state or inflammatory conditions, which are explored for the specific treatment of various diseases, such as cancer, autoimmunity or transplant rejection. Here, the impact of PE coating of ferumoxytol for uptake into both inflammatory and steady-state DCs and cellular responses to the MNP labeling is addressed. Labeling efficiency by uncoated and PE-coated ferumoxytol is highly variable in different DC subsets, and PE coating significantly improves the labeling of steady-state DCs. Uncoated ferumoxytol results in increased cytotoxicity of steady-state DCs after labeling that is abolished by the PE coating, while no increased cell death is observed in inflammatory DCs. Furthermore, uncoated and PE-coated ferumoxytol appears immunologically inert in inflammatory DCs but induces activation of steady-state DCs. These results show that PE coating of MNPs can be applied to endow particles with desired properties for enhanced uptake and cell type-specific responses in distinct target DC populations.
\end{abstract}




\section{Introduction}

Cellular immunotherapies become an increasingly promising approach for the development of integrative and personalized therapies. Antigen-presenting dendritic cells (DCs) are considered as particularly well suited for the development of such therapies due to their unique capacity to initiate and orchestrate antigenspecific immune responses [1]. DCs are the first cells to be involved in antigen sensing and scavenging, followed by processing and subsequent antigen-specific T-cell priming. Additionally, DCs can activate further immune effector cells, including B cells, natural killer (NK) cells and NKT cells [2]. Thus, DCs harmonize immune responses that eventually result in resistance to foreign pathogens and tolerance to self. Novel DC-based therapies, therefore, aim at establishing beneficial immune conditions in diseases such as cancer, chronic inflammation, autoimmunity, or transplant rejection. This is readily achieved using DCtargeted vaccines, mainly to induce anti-tumor immunity, while tolerogenic DCs are explored to silence autotoxic immune responses [3-6].

In vivo monitoring of engraftment, position and/or migration to the target site and function of transplanted cells could decisively contribute to the success of such therapies. Magnetic resonance imaging (MRI) of contrast agent-labeled cells, including DCs, has emerged as a well-suited imaging technique for tracking cells in vivo [7]. An outstanding feature of MRI is its capacity for long-term tracking of cells and their and migration while achieving excellent high-resolution images of target tissue in a three-dimensional anatomical context. Stable labeling of DCs with iron oxide-based magnetic nanoparticles (MNPs) as contrast agents has proven successful for MRI-based detection of cell deposits and their migration [8-12].

Ferumoxytol is an FDA approved iron oxide-based MNP formulation currently used as a drug to treat irondeficiency anemia in chronic kidney disease [13]. Ferumoxytol has also been used as a macrophageimaging agent as well as a blood-pool agent with MRI [14]. However, ferumoxytol alone did not result in effective cell labeling $[15,16]$. Thus, in previous studies, we successfully established conditions that enabled us to manufacture colloidal stable ferumoxytol particles coated with polyelectrolytes (PE), resulting in enhanced cell labeling [16]. In the present study, we mainly focused on the impact of PE-coated ferumoxytol MNPs on the labeling of different DC subsets, including inflammatory DCs, and steady-state conventional DCs (cDCs) and plasmacytoid DCs (pDCs) [17]. DCs generated in vitro from monocytes or CD34+ hematopoietic stem/progenitor cells (HSC) of blood or bone marrow using the granulocyte-macrophage colony-stimulating factor (GM-CSF) resemble a DC subset that occurs in vivo only under inflammatory conditions and thus referred to as inflammatory DCs [17]. Such patient-derived DC represents the prevailing DC subtype used in autologous cell-based immunotherapies so far [6]. However, employing primary existing cDCs and pDCs subtypes under steady-state conditions for therapeutic approaches are considered as potentially better-suited alternatives [3]. Here, we investigated the cellular intake of PE-coated and unmodified ferumoxytol into inflammatory DCs, and steady-state $\mathrm{CDCs}$ and $\mathrm{pDCs}$ generated in vitro cultures from mouse bone marrow. We assessed the impact of the PE coatings on cell viability, labeling efficiency, 
and intracellular iron content of MNP-labeled cells. Furthermore, immunophenotypic alterations of the various DC subtypes upon MNP incorporation were addressed. In summary, the results reported here revealed a differential impact of PE-coated and uncoated MNPs upon uptake in inflammatory DCs and steady-state DC, respectively.

\section{Materials and Methods}

\section{Polyelectrolyte coating of ferumoxytol}

The FDA approved iron oxide nanoparticle formulation ferumoxytol (Rienso®) was purchased from Takeda Pharma (Konstanz, Germany). High MW polyethyleneimine (PEI, 750 kDa) and low MW polydiallyldimethylammoniumchlorid (PDADMAC, 100-200 kDa) were obtained from Sigma-Aldrich (Taufkirchen, Germany). The surface coating of ferumoxytol was carried out using the LbL technique to deposit polyelectrolyte layers (Figure 1 A) [16,18-20]. The negatively charged ferumoxytol MNPs were added to an aqueous solution of the positively charged polyelectrolytes to prepare the coating layer. For coating with $\mathrm{PEl}, 3 \mathrm{mg}$ of ferumoxytol MNPs were added into $5 \mathrm{~mL}$ of a $1 \mathrm{~g} / \mathrm{L}$ PEl solution. For coating with PDADMAC, $9 \mathrm{mg}$ of ferumoxytol MNPs were added into $5 \mathrm{~mL}$ of a $2 \mathrm{~g} / \mathrm{L}$ PDADMAC solution [16]. After overnight shaking, the products were separated from the excess polyelectrolyte by magnetic separation and rinsed 3 times with double distilled water $(\mathrm{ddH} 2 \mathrm{O})$. Hydrodynamic diameter $(\mathrm{dH})$ of MNPs was obtained from dynamic light scattering (DLS) by cumulant fits using a Zetasizer 3000HSA (Malvern Instruments, Malvern, UK), which also provides the zeta potentials of the particles; each value reported is an average of at least ten consistent measurements. Uncoated and coated MNPs were visualized by transmission electron microscopy (TEM).

\section{Dendritic cell culture}

Various DC subsets were differentiated from hematopoietic DC progenitors of bone marrow suspensions from C57BL/6 mice (Charles River; Sulzfeld, Germany) as previously described (Figure 1B) [21,22]. In brief, for the generation of the inflammatory type of DCs, FIt3+ DC progenitors were obtained from mouse bone marrow cells after 7 days of culture in RPMI 1640 medium supplemented with $10 \%$ fetal calf serum, $2 \mathrm{mM}$ L-glutamine, $100 \mathrm{U} / \mathrm{ml}$ penicillin/streptomycin, and $50 \mu \mathrm{M} \beta$-mercaptoethanol (all from Life Technologies, Darmstadt, Germany) containing recombinant murine SCF (100 ng/ml), 25 ng/ml Flt3-ligand (Flt3L; PeproTech, Hamburg, Germany), $5 \mathrm{ng} / \mathrm{ml}$ hyper IL-6 (a kind gift from Dr. S. Rose-John, University of Kiel, Kiel, Germany), 40 ng/ml recombinant long-range IGF-1, $20 \mathrm{U} / \mathrm{ml}$ recombinant mouse GM-CSF, and 10-6 $\mathrm{M}$ dexamethasone (Sigma-Aldrich). After 7 days, differentiation of DC progenitors into inflammatory DCs was induced in culture medium supplemented with $200 \mathrm{U} / \mathrm{ml}$ recombinant murine GM-CSF [21]. 
A fraction of mouse bone marrow cells were cultivated for 7 days as described above to obtain steady-state $\mathrm{cDCs}$ and pDCs but in the absence of GM-CSF and dexamethasone [22]. After 7 days, such DC progenitors were differentiated into cDCs and pDCs using $50 \mathrm{ng} / \mathrm{ml} \mathrm{Flt3L}$ only.

Cell numbers were determined with an electronic cell counting device (CASY1, Roche, Penzberg, Germany). Mice were maintained under specific pathogen-free conditions in the central animal facility of the RWTH University Hospital Aachen. All animal experiments were approved by local authorities in compliance with the German animal protection law and EU guidelines (2010/63/EU) for animal protection.

\section{Flow cytometry}

Flow cytometry was used to analyze the DC phenotype and the effect of labeling as previously described [18]. Surface antigen expression on DC progenitors and DCs was examined using the following antibodies: Pacific Blue (PB)-conjugated anti-CD11b (clone M1/70), phycoerythrin-cyanin 7 (PE-Cy7) or fluorescein isothiocyanate (FITC)-conjugated anti-CD11C (N418), Allophycocyanin (APC)-conjugated anti-CD115 (AFS98), PE-Cy7-conjugated anti-CD117 (ACK), PE-conjugated anti-CD135 (A2F10), peridinin chlorophyll protein-cyanin 5.5. (PerCP-Cy5.5)-conjugated anti-B220 (RA3-6B2) APC-conjugated anti-MHC class II (MHC II; clone M5/114.15.2), and APC-conjugated anti-SiglecH (eBio440c) were purchased from eBioscience (San Diego, CA). PE-conjugated anti-CD24 (M1/69), FITC-conjugated anti-MHC II (2G9), and PerCP-Cy5.5-conjugated anti-Gr1 (RB6-8C5) were obtained from BD Biosciences (Heidelberg, Germany). Respective isotype controls were from BD Biosciences or eBioscience. Stained cells were analyzed with FACS Canto II flow cytometer (BD Bioscience), and data were evaluated using FlowJo software (TreeStar, Ashland, OR).

\section{MNP-labeling of DCs}

Sterile solutions of ferumoxytol MNPs were sonicated for $30 \mathrm{~min}$ prior to cell labeling and used with a final iron concentration of $10 \mu \mathrm{g} / \mathrm{ml}$. GM-DCs and FL-DCs were seeded at $2 \times 10^{6}$ cells $/ \mathrm{ml}$ and incubated with MNPs for $24 \mathrm{~h}$. Cells were harvested and washed in a PBS solution containing $2 \%$ FCS before being subjected to magnetic separation (MACS; Miltenyi Biotech, Bergisch Gladbach, Germany) as previously reported $[9,16]$. The labeling efficiency was calculated after the magnetic separation of the retained cell (Equation 1). Cell numbers of retained and not-retained cells were determined using CASY1 cell counter.

Labeling efficiency $(\%)=$ Retained Cell Number $/$ Total Cell Number $x 100$

(Equation 1)

\section{Cell viability assessment after MNP-labeling}

Cytotoxicity of PE-coated and uncoated MNPs on various DC subsets was evaluated using the Zombie Aqua Viability Kit (BioLegend, Fell, Germany) according to manufacturer' s protocol. Briefly, $5 \times 10^{5}$ cells 
after MNP-labeling were incubated in a 1:100 (v/v) dilution of the Zombie Aqua dye in a total volume of $50 \mu \mathrm{l}$ PBS for $20 \mathrm{~min}$ at room temperature in the dark. After washing with PBS, cells were analyzed with the FACSCanto II flow cytometer and data were evaluated using FlowJo software.

\section{Quantification of cellular iron content}

Intracellular iron concentration was determined using colorimetric ferrozine iron assay as previously described $[9,16]$. Briefly, $1 \times 10^{6}$ cells were lysed in $100 \mu 50 \mathrm{mM} \mathrm{NaOH}$ and ascorbic acid was used to reduce $\mathrm{Fe}^{3+}$ to $\mathrm{Fe}^{2+}$ ions that form a chelate complex with ferrozine (3-(2-pyridyl)-5,6-bis(phenyl sulfonic acid)-1,2,4-triazine; Sigma-Aldrich). The absorbance of Fe ${ }^{2+}$-ferrozine was measured at $550 \mathrm{~nm}$ using a FLUOStar OPTIMA plate reader (BMG Labtech, Ortenberg, Germany) and compared to the absorbance of $\mathrm{FeCl}_{3}$ standards.

\section{Prussian Blue staining of intracellular iron}

$1 \times 10^{5}$ cells labeled with MNPs were centrifuged onto a glass slide using a cytospin centrifuge (Thermo Fisher Scientific, Dreieich, Germany) to visualize the total iron uptake of cells. After washing in ddH2O, glass slides were subjected to Prussian Blue staining using a $1: 1$ solution $(\mathrm{v} / \mathrm{v})$ of $10 \% \mathrm{~K}_{4}\left[\mathrm{Fe}(\mathrm{CN})_{6}\right]$ (SigmaAldrich) and $20 \% \mathrm{HCl}$ for $20 \mathrm{~min}$. After washing in ddH2O, glass slides were counter-stained with Neutral Red dye (Roth, Karlsruhe, Germany) and mounted with coverslips using mounting medium (Dako, Hamburg, Germany). Sample images were obtained using the Leica DM6000B microscope and Diskus acquisition software (Hilgers, Koenigswinter, Germany).

\section{Transmission electron microscopy}

Transmission electron microscopy (TEM) images were obtained from $1 \times 10^{6}$ magnetically sorted DCs. Cells were fixed with $3 \%(\mathrm{w} / \mathrm{v})$ glutaraldehyde and embedded in $2 \%$ agarose. Samples were stained with $\mathrm{OsO}_{4}$, embedded in Epon and cut into $70 \mathrm{~nm}$ thick slices. Samples were analyzed without further contrast enhancement using a Philips EM 400T electron microscope at $60 \mathrm{kV}$ equipped with a CCD camera (MORADA, Olympus, Hamburg, Germany). TEM images were analyzed using NIH ImageJ software.

\section{Statistical analysis}

Numerical data were analyzed for significance by one-tailed Student's $t$ test with GraphPad Prism software (GraphPad, La Jolla, CA). A p-value below 0.05 was considered significant. 


\section{Results}

\section{Characterization of PE-coated ferumoxytol MNPs}

MNPs for biomedical applications generally possess a core-shell structure, whereby the shell surface is a crucial factor to impart good colloidal stability and biocompatibility, and ideally provides a scaffold for further functionalization. Ferumoxytol is a colloidal stable nanoparticle formulation with a core size of $6 \mathrm{~nm}$ and a polyglucose sorbitol carboxymethylether shell. We determined for ferumoxytol a hydrodynamic diameter of $29 \mathrm{~nm}$ and a $\zeta$-potential of - $44 \mathrm{mV}$ (Figure $1 \mathrm{~A}$ and B). We frequently use layer-by-layer (LbL) assembly of high and low molecular weight (MW) polyelectrolytes around MNPs to improve cellular responses, including uptake, intracellular localization and processing of MNPs, and thus on MRI properties of labeled cells $[16,18]$.

Here, based on previously established conditions for PE-coating of ferumoxytol, we used positively charged low MW polydiallyldimethylammonium chlorid (PDADMAC) and high MW polyethyleneimine (PEI) for coating (Figure S1A). LbL assembly of PDADMAC and PEI on ferumoxytol resulted in MNPs with the increased hydrodynamic diameter and positive surface charge, indicating successful PE coating (Figure $1 \mathrm{~A}$ and $B$ ). TEM imaging revealed that ferumoxytol MNPs aggregated upon PE-coating to larger size clusters (Figure 1C). Under the chosen conditions for coating, the low MW PDADMAC-coated ferumoxytol MNPs (referred in the following as + PDADMAC) and high MW PEl-coated ferumoxytol particles (mentioned in the following as $+\mathrm{PEI}$ ) were most similar in respect to surface charge, hydrodynamic diameter, and cluster size (Figure 1) and were therefore selected for further cell labeling studies.

\section{Differentiation of steady-state and inflammatory DC subsets}

A number of protocols have been developed for generating DCs in vitro from bone marrow or cord bloodderived HSC or blood monocytes. Our group has successfully established two-step culture systems that allow the amplification of fms related tyrosine kinase 3 positive (Flt3+) DC progenitor populations in the first step under growth-promoting conditions, which in a second step can be differentiated into fully functional DCs and thus yield high cell numbers [21,22]. Dependent on the amplification and differentiation condition that contains or lacks GM-CSF different DC subsets, including inflammatory DCs, as well as CDCs and pDCs, were generated.

Expression of the surface integrin CD11c represents a hallmark of fully differentiated DCs, whereas Flt3+ progenitors are CD11C negative but express the integrin CD11b (Figure 2 and Figure S1A). After 7 days under proliferating conditions, the differentiation of Flt3+ progenitors towards DCs was induced (referred to as day 0 in Figure $2 \mathrm{~A}$ and Figure S1A) using either GM-CSF or Flt3 ligand (FIt3L), respectively. The Flt3+ progenitor grown in the presence of GM-CSF and dexamethasone readily differentiates within 6 days into fully functional DCs following administration of GM-CSF only (hereinafter referred to as GM-DCs). The majority of GM-DCs displayed a low to an intermediate expression of Major Histocompatibility Complex 
class II (MHC II) molecules on the cell surface (Figure 2A), which was indicative for an immature developmental stage of GM-DCs [21].

A modified two-step protocol was used that lacks GM-CSF and dexamethasone to obtain steady-state cDCs and pDCs [22]. This approach recapitulated more closely the in vivo DC development from a multipotent hematopoietic progenitor (MPP) through a common DC-restricted progenitor (CDP) intermediate stage [23]. Accordingly, under growing conditions, the DC progenitor populations were more heterogeneous reflected by different surface marker profiles: MPPs express high levels of c-kit (CD117) but are low to negative for FIt3 (CD135), while CDPs express c-kit at low levels and Flt3 at high levels (Figure S1A). CDPs also express CD11b and the macrophage-colony stimulating factor receptor (M-CSFR, CD115) at higher levels than MPPs (Figure S1A). Collectively, the in vitro amplified CDPs resembled a phenotype similar to their in vivo counterparts $[24,25]$. The modified growing conditions allowed differentiation of Flt3+ DC progenitors using FIt3L only that resulted in concurrent differentiation of both $\mathrm{cDCs}$ and pDCs within 6 days (Figure 2B and Figure S1A and B). Differentiated CDCs and pDCs exhibited an immature phenotype that resembles steadystate DC subsets in lymphoid tissues, such as spleen or lymph nodes [22]. In the bulk culture, we refer to both DC subsets in the following as FL-DCs. Mouse pDCs expressed CD11c, SiglecH, and B220 and downregulated CD11b expression, while cDCs expressed CD11c, CD11b and were negative for SiglecH and B220 (Figure 2B and Figure S1A, B). Moreover, both steady-state DC populations displayed a clearly distinct MHC II expression profile with definite MHC II expression in CDCs and the absence of MHC II expression in pDCs (Figure 2B) that is in line with the surface marker profile of their in vivo equivalents [26].

\section{Labeling of DCs with uncoated and PE-coated ferumoxytol}

Next, MNP interaction with inflammatory and steady-state DCs was investigated. After incubation of cells with uncoated and PE-coated ferumoxytol particles for $24 \mathrm{~h}$, the cells were subjected to magnetic sorting to separate MNP-labeled DCs and unlabeled DCs and thus to quantify the efficiency of MNP uptake. The labeling efficiency was calculated with the formula given in Equation 1, and the results are shown in Figure 3A. Labeling efficiency by uncoated ferumoxytol and PE-coated MNPs was found highly variable in GMDCs and FL-DCs (Figure 3A). Uncoated ferumoxytol resulted in the labeling of only $20 \%$ of FL-DCs in agreement with previously reported poor labeling efficiency $[15,16]$. In contrast, GM-DCs showed significantly higher labeling efficiency by uncoated ferumoxytol than FL-DCs. The PE-coating of MNPs resulted in increased cell labeling in line with previous studies but with different impact [16,27]. For FL-DCs, the MNP coating with both PEI and PDADMAC resulted in a four-fold higher labeling of FL-DCs than with uncoated MNPs (Figure 3A). In contrast, in GM-DCs, only the MNP-coating using PDADMAC improved MNP uptake by the cells, while PEI-coated MNPs showed no significantly improved labeling compared to uncoated MNPs. These results indicate that the improved labeling of GM-DCs by PDADMAC-coated ferumoxytol cannot be attributed solely to the positive surface charge. However, charge reversal by the PEcoating might indeed represent the primary cause for the improved uptake of MNP by FL-DCs. Taken 
together, the data suggest that different uptake mechanisms for MNPs are active in inflammatory and steady-state DCs, respectively.

The different MNP surface coatings and/or uptake mechanisms may impact on the intracellular loading of MNPs. To address this, we quantified the cellular iron content upon uptake of MNPs using a ferrozine-based colorimetric assay (Figure 3B) and visualized the MNP loading by Prussian blue staining (Figure $3 \mathrm{C}$ ). It was found that GM-DCs were capable of taking up $15 \pm 5.3 \mathrm{pg}$ iron/cell with uncoated ferumoxytol particles, 12 $\pm 1.8 \mathrm{pg}$ iron/cell with PDADMAC-coated MNPs and $12.8 \pm 1.5 \mathrm{pg}$ iron/cell with PEl-coated MNPs. The iron concentrations in FL-DCs were $8.7 \pm 0.6 \mathrm{pg}$ iron/cell with uncoated ferumoxytol particles, $9.7 \pm 1.4 \mathrm{pg}$ iron/cell with PDADMAC-coated ferumoxytol particles and $12.2 \pm 2.2 \mathrm{pg}$ iron/cell with PEl-coated ferumoxytol particles. No significant differences in the amount of intracellular iron were observed between GM-DCs and FL-DCs when PE-coated ferumoxytol particles were used. However, the internalized iron content was significantly higher in GM-DCs than in FL-DCs when labeled with uncoated ferumoxytol (Figure 3B). Notably, the increased labeling efficiency of FL-DCs with PE-coated MNPs was not reflected by elevated iron concentrations indicating a constraint in the uptake capacity.

The ascertained intracellular iron concentrations represent average values over all magnetically separated and hence labeled cells. Thus, the intracellular iron load was additionally visualized using Prussian blue staining to confirm the uptake of MNPs in labeled cells (Figure 3C). Iron deposits stained by Prussian blue showed a variable distribution of internalized MNPs in retained cells and revealed differentially sized agglomerates of the different MNPs within cells (Figure $3 \mathrm{C}$ ). Furthermore, we did not observe any staining of iron in not-retained cells, confirming the absence of MNPs.

Labeling of steady-state DCs, including both cDCs and pDCs subpopulations and selection of labeled cells by magnetic sorting, was performed in bulk cultures. However, the analysis of cells after sorting by flow cytometry allowed discriminating labeled cDCs from labeled pDCs and to further address DC populationspecific cellular responses to MNP uptake. Both $\mathrm{CDC}$ and $\mathrm{pDC}$ populations were identified according to differential CD11b and SiglecH expression (Figure 2B and Figure 4A). After 6 days of differentiation, around $30 \%$ of $C D 11 c+$ cells were identified as $\mathrm{CDCs}$, and $50 \%$ were $\mathrm{pDCs}$, while the other CD11c+ cells could not be clearly assigned to a specific subset (Figure $2 \mathrm{~B}$ ). A comparable ratio of $27.5 \% \pm 1.8 \%$ (mean value \pm SEM; $n=3$ ) cDCs and $49.0 \% \pm 9.0 \%$ pDCs was also observed after further $24 \mathrm{~h}$ of culture (Figure $4 \mathrm{~A}, \mathrm{~B}$ ). The composition of FL-DCs after MNP-labeling for $24 \mathrm{~h}$ and magnetic separation was assessed accordingly (Figure 4A, B). After labeling with uncoated ferumoxytol, the ratio of $\mathrm{cDCs}$ and $\mathrm{pDC}$ changed towards a higher proportion of labeled cDCs $(52.6 \% \pm 12.1 \%$ vs. $34.8 \% \pm 10 \%$ of labeled $\mathrm{pDCs})$ that further points to the poor labeling efficiency of uncoated ferumoxytol especially of pDCs (Figure 4C). Labeling with PDADMAC-coated MNPs was found equally efficient in both $\mathrm{CDCs}$ and $\mathrm{pDCs}$ as displayed by similar ratios as in unlabeled controls $(25.8 \% \pm 2.5 \% \mathrm{cDCs}$ and $55.5 \% \pm 5.1 \% \mathrm{pDCs})$. Labeling with PEI-coated MNPs resulted in a slight shift in the ratio of $\mathrm{CDCs}$ and $\mathrm{pDCs}$ towards labeled pDCs, demonstrating that PEl-coated MNPs are most effective for labeling of pDCs (Figure 4C). 


\section{Cytotoxicity assessment of uncoated and PE-coated MNPs upon uptake into DCs}

We next examined whether MNPs affect cell viability of DCs after MNP-uptake by subjecting MNP-labeled and unlabeled cells to Zombie Aqua staining for detection of dead cells using flow cytometry (Figure 5). Unlabeled negative control GM-DCs showed a contingent of $5.2 \%$ of dead cells (Figure 5A). Unlabeled GMDCs were stimulated with the bacterial wall component lipopolysaccharide (LPS) that leads to prominent DC activation [18] and 24 hours after LPS treatment, 9.6\% of GM-DCs was found dead. After labeling GMDCs with MNPs for $24 \mathrm{~h}$ and magnetic sorting the proportion of dead cells detected was $4.8 \%$ for labeling with uncoated ferumoxytol, $5.1 \%$ for labeling with PDADMAC-coated ferumoxytol, and $4.6 \%$ with PEIcoated ferumoxytol. Thus, labeling of GM-DCs using uncoated and PE-coated ferumoxytol particles did not result in adverse cytotoxic effects at the concentration used here for labeling. Remarkably, particle size and surface charge also revealed no impact on cytotoxicity in GM-DCs in line with results from our previous studies $[16,18]$.

In contrast to inflammatory GM-DCs, the response of steady-state FL-DCs to MNP labeling revealed a differential impact of uncoated and PE-coated ferumoxytol on cell viability after labeling (Figure 5B and C). Notably, the level of dead cells detectable in untreated and LPS stimulated FL-DCs was found lower compared to GM-DCs. In untreated $\mathrm{CDC}$ and $\mathrm{pDC}$ populations, $2.5 \%$ and $0.5 \%$ of cells, respectively, were determined as dead cells. Furthermore, stimulation of FL-DCs with LPS, unlike GM-DCs, did not result in an increased number of dead cells, although it led to effective DC activation (see below 3.5. and Figure 6C). However, the labeling of FL-DCs with uncoated ferumoxytol resulted in an approximately 3- to 5-fold increase in the number of dead cells in cDCs $(11.6 \% \pm 4.4 \%$, mean value \pm SD; $n=3)$ and $p D C s(1.7 \% \pm$ $0.4 \%$ ) yet on an overall low level. In contrast, the proportion of dead $\mathrm{cDCs}$ and $\mathrm{pDCs}$ after labeling with PDADMAC-coated MNPs ( $2.6 \%$ and $0.5 \%$, respectively) or PEl-coated MNPs ( $2.5 \%$ and $0.8 \%$, respectively) were not significantly changed compared to untreated FL-DCs. Thus, the PE-coating abolished the harmful impact of ferumoxytol in CDCs and pDCs, while yielding higher labeling rates and intracellular iron concentrations in these steady-state DCs.

Immunophenotypic differences in steady-state and inflammatory DCs upon MNP-labeling

According to their specific antigen-uptake and -presentation function, DCs are well equipped with pattern recognition receptors that allow them to sense and uptake a wide range of antigens [28]. DC activation in response to foreign antigens leads to the maturation of DCs from their immature state that includes upregulation of $\mathrm{MHC}$ II on the cell surface required for effective antigen presentation to T cells. Here, we now assessed the immune stimulatory capacity of uncoated and PE-coated ferumoxytol particles upon uptake into both steady-state and inflammatory DCs, respectively. To this end, GM-DCs and FL-DCs were analyzed for MHC II expression by flow cytometry upon exposure to MNPs for $24 \mathrm{~h}$ and magnetic separation 
(Figure 6). As a control for effective DC activation, DCs were stimulated with LPS for $24 \mathrm{~h}$. In the absence of MNPs, GM-DCs displayed low to intermediate expression levels of MHC II, which increased to high levels after stimulation with LPS (Figure 6A, B). Importantly, MNP-labeled GM-DCs showed MHC II expression profiles similar to profiles of untreated cells, indicating that exposure to and uptake of MNPs did not lead to activation of inflammatory DCs (Figure 6A, B) in line with our previous results [18]. In addition, the comparison of MNP-labeled with non-labeled cells (i.e., cells not magnetically retained) revealed enrichment of not terminally differentiated DCs (i.e., negative for CD11C and MHC II) in the not-retained fraction concurrent with the enhancement of terminally differentiated DCs in the MNP-labeled fraction (Figure 6A) that highlights the selective uptake capacity of DCs for MNPs.

In contrast to GM-DCs, the steady-state FL-DCs revealed a different MHC II expression profile with a more homogeneous expression of MHC II in cDCs at intermediate levels and the absence of MHC II expression in pDCs (Figure 3B and Figure 7C). LPS stimulation of FL-DCs showed a differential impact on FL-DC activation that led to the maturation of the entire $\mathrm{CDC}$ population to high-level $\mathrm{MHC}$ II expressing cells, while for pDCs, only a fraction of cells respond to LPS by upregulation of MHC II to intermediate levels (Figure 7C). Remarkably, and in contrast to inflammatory DCs, uptake of MNPs induced upregulation of surface MHC II expression in both cDCs and pDCs, although to a lesser extent than LPS (Figure 7D-F). Taken together, the results indicate that sensing and response to MNPs are differentially controlled in inflammatory DCs and steady-state cDCs and pDCs.

\section{Discussion}

Surface coating of MNPs using polyelectrolytes possess great potential to tailor MNP properties for their use in various biomedical applications, including cell labeling and tracking by MRI. In this study, we demonstrated that specific PE coatings caused different cellular responses in distinct DC subpopulations. We found a selective labeling capacity of PE-coated MNPs dependent on the DC subtype together with a differential impact on the cytotoxic as well as immunomodulatory consequences of both uncoated and PEcoated ferumoxytol.

Engineered iron oxide MNPs are increasingly harnessed as advanced tools for medical applications, including cell labeling and tracking, targeted drug release, non-invasive monitoring of therapy, and vaccination $[14,29]$. Moreover, iron oxide-based MNPs are considered to be most suitable for combining a number of these applications into a single multifunctional formulation, thus providing an accurate nano theranostics tool.

Currently, ferumoxytol is the only FDA approved iron oxide-based MNP formulation initially launched for iron replacement therapy. It is recently investigated extensively as a contrast agent in MRI since it shows fewer side effects such as allergic or idiosyncratic reactions than other contrast agents [14]. Moreover, ferumoxytol does not entail a risk for the development of nephrogenic systemic fibrosis and thus may substitute 
gadolinium-based contrast agents as a blood pool agent in a number of MRI applications. Accordingly, attempts have been made to use ferumoxytol as a contrast agent for labeling and tracking of cells in vivo using MRI, including mesenchymal stromal cells, neural stem cells, and immune cells, including T cells, monocytes, and DCs $[10,24,27,30]$. The uptake by macrophages in vivo is being explored as a novel imaging approach for the assessment of lymph nodes, tumors, and vascular lesions. For example, in a preclinical model of autoimmune myocarditis, the iron oxide MNPs ingested by macrophages improved the distinction of areas of severe inflammation by MRI compared to conventional T2-weighted and gadoliniumenhanced MRI [31]. However, in a more recently published clinical study in patients with acute myocarditis, ferumoxytol-enhanced MRI was found unable to identify myocarditis by detection of macrophage activity [32]. As a possible explanation for the contradictory finding to the previous study, the authors discuss the limited uptake capacity of ferumoxytol by macrophages compared to other iron oxide MNPs. This is in line with previous studies that found no efficient labeling of cells with ferumoxytol alone or in combination with protamine $[10,15]$. However, a combination of ferumoxytol with protamine and heparin were described to result in improved labeling of neural stem cells, bone marrow stromal cell, monocytes, and $\mathrm{T}$ cells and an increase in T2 relaxivity compared to ferumoxytol alone [27].

We frequently use layer-by-layer ( $L b L$ ) assembly of polyelectrolytes for coating of MNPs to improve cellular responses, including uptake, intracellular localization and processing of MNPs, and thus on MRI properties of labeled cells. For example, coating of oleate-stabilized MNPs with PDADMAC resulted in a more dense agglomeration of MNPs within endosomal compartments of DCs, resulting in a larger magnetic susceptibility effect $\left(\mathrm{T}^{*}\right)$ when compared with loosely packed MNPs that were coated with polystyrene sulphonate or chitosan [18]. Noteworthy, the observed differences in MRI contrast-agent properties of PE-coated MNPs were not related to the total amount of iron taken up by cells, as chitosan-coated MNPs yielded the highest iron concentration in DCs, but exhibited inferior performance in MRI [18]. Here, we used the positively charged polyelectrolytes PEI and PDADMAC for the coating of ferumoxytol. PEI and PDADMAC have proven to be excellently biocompatible as PE coating for MNPs and allowed stable colloidal coating of ferumoxytol $[16,18]$. Remarkably, the PE coating of ferumoxytol has significantly improved the labeling of steady-state DC by up to 4 -fold. In contrast, uncoated ferumoxytol was already incorporated by $\sim 50 \%$ of the inflammatory GM-DCs, and the PE coating did not result in substantially improved cell labeling.

Patient-derived GM-DCs are to date the prevailing DC subtype used in autologous cell-based immunotherapy studies [6]. Another approach, however, is to harness the available steady-state cDCs and pDCs in vivo by direct targeting of specific subsets and to activate their subset specific properties depending on the type of disease [3]. Accordingly, attempts are made by, e.g., using specific antibodies that recognize and bind to unique subsets as carriers for antigens, drugs or immune regulatory factors [33]. Besides, very similar approaches have emerged using nano carrier-based delivery systems, including iron oxide-based MNPs [34]. A wide range of nanoscale materials has been developed that can serve as platforms for assembling various antigens, adjuvants, and other immunomodulatory reagents bound to the surface of and/or enveloped in such nanocarriers. Interestingly, a number of such antigen and adjuvant factors 
represent polyelectrolytes that target specific immune pathways. One such pathway is activated by toll-like receptors (TLRs), and TLR agonists are intensively explored as molecular adjuvants for vaccination. This includes negatively charged nucleoside analogs that act as specific TLR agonists, such as double-stranded RNA or the synthetic analog poly(I:C) for TLR3, bacterial or viral single-stranded RNA for TLR7 or unmethylated DNA oligonucleotides (ODN) containing CpG motifs for TLR9. For example, simultaneously applied poly(Y-glutamic acid)-based NPs loaded with a tumor model antigen (OVA) or with poly(I:C) induced higher anti-tumor activity as compared to the activity without NPs [35]. Even more elegantly, the group of Jewell et al. [36] used LbL-assembly of positively charged OVA peptide (SIINFEKL) and negatively charged poly $(\mathrm{l}: \mathrm{C})$ around calcium carbonate templates where the core was finally removed using a chelator to create hollow capsules. These immune polyelectrolyte multilayers (iPEMs) were able to activate steady-state DCs to a greater extent than dose-matched soluble factors alone [34].

The TLR specificity of agonists can be further exploited to target specific DC subsets since DC populations express non-overlapping sets of TLRs [28]. For example, in humans, TLR9 is expressed by pDCs but not inflammatory DCs, and thus CpG ODN acts particularly on pDCs to induce type I interferon (IFN) production.

The synthetic polyelectrolytes PEI and PDADMAC used in our study elicited a slight adjuvant effect in steady-state DCs similar to ferumoxytol alone but to a much lesser extent than the TLR4 agonist LPS. In contrast, all NP formulations were immunological inert in inflammatory DCs, suggesting that GM-DCs, unlike FL-DCs, lack the respective recognition receptors.

A hitherto unrecognized immunomodulatory activity of ferumoxytol has been described in a recent study on the mechanism of tumor growth inhibition by ferumoxytol [37]. While ferumoxytol showed no direct cytotoxic effects on tumor cells, the study by Zanganeh et al. [37] revealed that ferumoxytol expedited the recruitment of macrophages towards tumor cells and induced a phenotypic shift towards pro-inflammatory M1 polarization. Tumor-associated macrophages generally develop towards an M2-like phenotype at a later stage of tumor progression. At the same time, M1 polarization requires activation by canonical IRF/STAT signaling pathways activated by IFNs and TLR signaling [38]. Ferumoxytol then elicits the production of reactive oxygen species (ROS) via the Fenton reaction in M1 macrophages, thereby inducing the apoptosis of cancer cells [37]. It is tempting to speculate that ferumoxytol is recognized and acts via TLRs on macrophages, but the precise mechanism has not yet been addressed. According to our data, however, it appears unlikely that ferumoxytol is recognized by TLR4, which is highly expressed in macrophages as well as GM-DC. In our study, both PE coated and uncoated ferumoxytol did neither result in activation nor apoptosis induction of inflammatory DC, in contrast to stimulation with the TLR4 ligand LPS. In contrast, uncoated ferumoxytol increased apoptosis in steady state DCs probably by generation of ROS through the Fenton reaction. Thus, the differential impact of ferumoxytol on the cytotoxicity of GM-DCs and FL-DCs further points towards DC subset specific pathways activated in responses to the MNPs. 


\section{Conclusion}

LbL assembly of polyelectrolytes around MNPs represents a versatile means to tailor MNP surface properties. Here, we found that high MW PEI and low MW PDADMAC are well suitable for the coating of ferumoxytol to improve the cell-labeling efficacy of various DC subtypes. PE-coated ferumoxytol was essentially non-toxic to labeled cells. Moreover, PE coating diminished adverse cytotoxic effects of uncoated ferumoxytol in labeled steady-state DCs.

Polyelectrolyte coating of MNPs can be conceived to endow particle surfaces with desired properties. This can be employed, e.g. (i) for enhanced or even targeted uptake into cells, thereby augmenting their performance as contrast agents for $\mathrm{MRI}$ and (ii) to provide additional functionalities such as immunoregulatory activities. For clinical use, immunomodulatory activities provided by the MNP formulation may have beneficial effects, e.g., such as an adjuvant function in vaccination applications. However, the careful assessment of such immunomodulatory activities is inevitable to avoid deleterious side effects of MNPs beyond cytotoxicity. The specific antigen-uptake and -presentation, as well as immune regulatory function of distinct DC subpopulations, makes them particularly important for the study of such MNP properties. Depending on the molecular pattern of the MNP shell, specific DC subsets - as shown here can be expected to recognize MNPs as potentially foreign antigens, therefore providing the capability to biologically sense MNP shell chemistry. This can lay the foundation for further functionalization of MNPs that will combine efficient labeling of DCs for cell tracking with additional activities that impact on DC function. In this respect, the combination of ferumoxytol coated with clinically approved polyelectrolytes should expedite a faster translation of this approach to clinical use.

\section{Acknowledgements}

We would like to thank H. Koenigs, RWTH Aachen University Hospital, Department of Pathology, for assistance with electron microscopy. This work was supported by the Flow Cytometry Facility, a core facility of the Interdisciplinary Center for Clinical Research (IZKF) Aachen within the Faculty of Medicine at RWTH Aachen University. Part of this work was supported by the Excellence Initiative of the German federal and state governments (ERS OPSP006 to J. E. W. and T. H.) by RWTH Aachen University, Germany. T. H. received a grant from the START program of the Faculty of Medicine, RWTH Aachen University. N. C. thanks the CEMACUBE program (Erasmus Mundus, Action 1, Common European Master's course in Biomedical Engineering) for providing the scholarship.

\section{References}

[1] R.M. Steinman, J. Banchereau, Taking dendritic cells into medicine, Nature. 449 (2007) 419-426.

[2] J. Banchereau, F. Briere, C. Caux, J. Davoust, S. Lebecque, Y.-J. Liu, B. Pulendran, K. Palucka, Immunobiology of dendritic cells, Annu. Rev. Immunol. 18 (2000) 767-811. 
[3] K. Palucka, J. Banchereau, Dendritic-cell-based therapeutic cancer vaccines, Immunity. 39 (2013) 38-48.

[4] G. Beriou, A. Moreau, M.C. Cuturi, Tolerogenic dendritic cells: applications for solid organ transplantation, Curr. Opin. Organ Transplant. 17 (2012) 42-47.

[5] C.M.U.U. Hilkens, J.D. Isaacs, Tolerogenic dendritic cell therapy for rheumatoid arthritis: where are we now?, Clin. Exp. Immunol. 172 (2013) 148-157.

[6] J. Sprooten, J. Ceusters, A. Coosemans, P. Agostinis, S. De Vleeschouwer, L. Zitvogel, G. Kroemer, L. Galluzzi, A.D. Garg, Trial watch: dendritic cell vaccination for cancer immunotherapy, Oncoimmunology. 8 (2019) e1638212.

[7] E.T. Ahrens, J.W.M. Bulte, Tracking immune cells in vivo using magnetic resonance imaging, Nat. Rev. Immunol. 13 (2013) 755-763.

[8] I.J.M. De Vries, W.J. Lesterhuis, J.O. Barentsz, P. Verdijk, J.H. van Krieken, O.C. Boerman, W.J.G. Oyen, J.J. Bonenkamp, J.B. Boezeman, G.J. Adema, J.W.M. Bulte, T.W.J. Scheenen, C.J. a Punt, A. Heerschap, C.G. Figdor, Magnetic resonance tracking of dendritic cells in melanoma patients for monitoring of cellular therapy., Nat. Biotechnol. 23 (2005) 1407-1413.

[9] S. Schwarz, F. Fernandes, L. Sanroman, M. Hodenius, C. Lang, U. Himmelreich, T. Schmitz-Rode, D. Schueler, M. Hoehn, M. Zenke, T. Hieronymus, Synthetic and biogenic magnetite nanoparticles for tracking of stem cells and dendritic cells, J. Magn. Magn. Mater. 321 (2009) 1533-1538.

[10] G.A. Dekaban, A.M. Hamilton, C.A. Fink, B. Au, S.N. de Chickera, E.J. Ribot, P.J. Foster, Tracking and evaluation of dendritic cell migration by cellular magnetic resonance imaging, Wiley Interdiscip. Rev. Nanomedicine Nanobiotechnology. 5 (2013) 469-483.

[11] Y. Xu, C. Wu, W. Zhu, C. Xia, D. Wang, H. Zhang, J. Wu, G. Lin, B. Wu, Q. Gong, B. Song, H. Ai, Superparamagnetic MRI probes for invivo tracking of dendritic cell migration with a clinical 3T scanner, Biomaterials. 58 (2015) 63-71.

[12] Z. Zhang, W. Li, D. Procissi, K. Li, A.Y. Sheu, A.C. Gordon, Y. Guo, K. Khazaie, Y. Huan, G. Han, Antigen-loaded dendritic cell migration: MR imaging in a pancreatic carcinoma model, Radiology. 274 (2015) 192-200.

[13] P.L. McCormack, Tranexamic acid, Drugs. 72 (2012) 585-617.

[14] M.R. Bashir, L. Bhatti, D. Marin, R.C. Nelson, Emerging applications for ferumoxytol as a contrast agent in MRI, J. Magn. Reson. Imaging. 41 (2015) 884-898.

[15] Y.J. Wu, L.L. Muldoon, C. Varallyay, S. Markwardt, R.E. Jones, E.A. Neuwelt, In vivo leukocyte labeling with intravenous ferumoxides/protamine sulfate complex and in vitro characterization for cellular magnetic resonance imaging, Am. J. Physiol. Physiol. 293 (2007) C1698-C1708.

[16] N. Celikkin, L. Jakubcová, M. Zenke, M. Hoss, J.E. Wong, T. Hieronymus, Polyelectrolyte coating of ferumoxytol nanoparticles for labeling of dendritic cells, J. Magn. Magn. Mater. 380 (2015) 3945.

[17] K. Shortman, S.H. Naik, Steady-state and inflammatory dendritic-cell development, Nat Rev Immunol. 7 (2007) 19-30.

[18] S. Schwarz, J.E. Wong, J. Bornemann, M. Hodenius, U. Himmelreich, W. Richtering, M. Hoehn, M. Zenke, T. Hieronymus, Polyelectrolyte coating of iron oxide nanoparticles for MRI-based cell tracking., Nanomedicine. 8 (2012) 682-691.

[19] J.E. Wong, W. Richtering, Surface Modification of Thermoresponsive Microgels via Layer-by-Layer Assembly of Polyelectrolyte Multilayers, Progr Colloid Polym Sci. (2006) 45-51.

[20] J.E. Wong, A. Krishnakumar Gaharwar, D. Müller-Schulte, D. Bahadur, W. Richtering, A.K. Gaharwar, D. Müller-Schulte, D. Bahadur, W. Richtering, Layer-by-layer assembly of a magnetic 
nanoparticle shell on a thermoresponsive microgel core, J. Magn. Magn. Mater. 311 (2007) 219223.

[21] T. Hieronymus, T.C. Gust, R.D. Kirsch, T. Jorgas, G. Blendinger, M. Goncharenko, K. Supplitt, S. Rose-John, A.M. Müller, M. Zenke, Progressive and Controlled Development of Mouse Dendritic Cells from Flt3+CD11b+ Progenitors In Vitro, J. Immunol. 174 (2005) 2552-2562.

[22] P. Felker, K. Seré, Q. Lin, C. Becker, M. Hristov, T. Hieronymus, M. Zenke, TGF-beta1 accelerates dendritic cell differentiation from common dendritic cell progenitors and directs subset specification toward conventional dendritic cells., J. Immunol. 185 (2010) 5326-5335.

[23] M.A. Schmid, D. Kingston, S. Boddupalli, M.G. Manz, Instructive cytokine signals in dendritic cell lineage commitment, Immunol. Rev. 234 (2010) 32-44.

[24] S.H. Naik, P. Sathe, H.-Y. Park, D. Metcalf, A.I. Proietto, A. Dakic, S. Carotta, M. O’Keeffe, M. Bahlo, A. Papenfuss, Development of plasmacytoid and conventional dendritic cell subtypes from single precursor cells derived in vitro and in vivo, Nat. Immunol. 8 (2007) 1217-1226.

[25] N. Onai, A. Obata-Onai, M.A. Schmid, T. Ohteki, D. Jarrossay, M.G. Manz, Identification of clonogenic common Flt3+ M-CSFR+ plasmacytoid and conventional dendritic cell progenitors in mouse bone marrow, Nat. Immunol. 8 (2007) 1207-1216.

[26] M. Merad, M.G. Manz, Dendritic cell homeostasis, Blood, J. Am. Soc. Hematol. 113 (2009) 34183427.

[27] M.S. Thu, L.H. Bryant, T. Coppola, E.K. Jordan, M.D. Budde, B.K. Lewis, A. Chaudhry, J. Ren, N.R.S. Varma, A.S. Arbab, J.A. Frank, Self-assembling nanocomplexes by combining ferumoxytol, heparin and protamine for cell tracking by magnetic resonance imaging., Nat. Med. 18 (2012) 4637.

[28] A. Iwasaki, R. Medzhitov, Toll-like receptor control of the adaptive immune responses, Nat. Immunol. 5 (2004) 987-995.

[29] V.F. Cardoso, A. Francesko, C. Ribeiro, M. Bañobre-López, P. Martins, S. Lanceros-Mendez, Advances in magnetic nanoparticles for biomedical applications, Adv. Healthc. Mater. 7 (2018) doi: 10.1002/adhm.201700845.

[30] A. Khurana, H. Nejadnik, F. Chapelin, O. Lenkov, R. Gawande, S. Lee, S.N. Gupta, N. Aflakian, N. Derugin, S. Messing, Ferumoxytol: a new, clinically applicable label for stem-cell tracking in arthritic joints with MRI, Nanomedicine. 8 (2013) 1969-1983.

[31] H. Moon, H.E. Park, J. Kang, H. Lee, C. Cheong, Y.T. Lim, S.-H. Ihm, K.-B. Seung, F.A. Jaffer, J. Narula, Noninvasive assessment of myocardial inflammation by cardiovascular magnetic resonance in a rat model of experimental autoimmune myocarditis, Circulation. 125 (2012) 26032612.

[32] C.G. Stirrat, S.R. Alam, T.J. MacGillivray, C.D. Gray, M.R. Dweck, K. Dibb, N. Spath, J.R. Payne, S.K. Prasad, R.S. Gardner, Ferumoxytol-enhanced magnetic resonance imaging in acute myocarditis, Heart. 104 (2018) 300-305.

[33] C.H.K. Lehmann, L. Heger, G.F. Heidkamp, A. Baranska, J.J. Lühr, A. Hoffmann, D. Dudziak, Direct delivery of antigens to dendritic cells via antibodies specific for endocytic receptors as a promising strategy for future therapies, Vaccines. 4 (2016) 8. doi: 10.3390/vaccines 4020008.

[34] S. Singha, K. Shao, K.K. Ellestad, Y. Yang, P. Santamaria, Nanoparticles for immune stimulation against infection, cancer, and autoimmunity, ACS Nano. 12 (2018) 10621-10635.

[35] S.-Y. Kim, Y.-W. Noh, T.H. Kang, J.-E. Kim, S. Kim, S.H. Um, D.-B. Oh, Y.-M. Park, Y.T. Lim, Synthetic vaccine nanoparticles target to lymph node triggering enhanced innate and adaptive antitumor immunity, Biomaterials. 130 (2017) 56-66. 
[36] M.L. Bookstaver, K.L. Hess, C.M. Jewell, Self-assembly of immune signals improves codelivery to antigen presenting cells and accelerates signal internalization, processing kinetics, and immune activation, Small. 14 (2018) e1802202.

[37] S. Zanganeh, G. Hutter, R. Spitler, O. Lenkov, M. Mahmoudi, A. Shaw, J.S. Pajarinen, H. Nejadnik, S. Goodman, M. Moseley, L.M. Coussens, H.E. Daldrup-Link, Iron oxide nanoparticles inhibit tumour growth by inducing pro-inflammatory macrophage polarization in tumour tissues, Nat. Nanotechnol. 11 (2016) 986-994.

[38] A. Sica, A. Mantovani, Macrophage plasticity and polarization: in vivo veritas, J. Clin. Invest. 122 (2012) 787-795. 


\section{Figure legends}

Figure 1. Characterization of uncoated, PDADMAC-, and PEI-coated ferumoxytol MNPs. (A) Hydrodynamic diameter of MNPs determined by DLS scans. (B) Surface charges (zeta potential) of MNPs obtained by Zetasizer measurements. (C) TEM images of (I) PDADMAC-coated ferumoxytol and (II) PEl-coated ferumoxytol clusters. Scale bars, $100 \mathrm{~nm}$.

Figure 2. Phenotypic characterization of developing GM-DCs and FL-DCs during differentiation from FIt+ DC progenitors. (A) GM-DC development was monitored for CD11b and CD11c expression (upper panel), and MHC II expression (blue lines; lower panel) on days 0, 3 and 6 of differentiation using GM-CSF. (B) FL-DC development towards cDCs and pDCs on days 3 and 6 of differentiation using Flt3L was examined by gating on $\mathrm{CD} 11 \mathrm{c}, \mathrm{CD} 11 \mathrm{~b}$, and SiglecH (left and middle panel). According to CD11b and SiglecH expression $\mathrm{CDC}$ and $\mathrm{pDC}$ populations were identified and $\mathrm{MHC}$ II expression within specific DC subsets is shown in the right panel (pDCs, red lines and boxes; cDCs, black lines and boxes). Gray histograms show isotype control staining. Representative flow cytometry data from 3-5 independent experiments are shown.

Figure 3. Uptake of uncoated and PE-coated ferumoxytol particles into DCs. Inflammatory DCs (GMDCs) and steady state DCs (FL-DCs) were incubated with the respective MNPs for $24 \mathrm{~h}$ and subjected to magnetic separation of MNP-loaded cells from unlabeled cells. (A) Labeling efficiency was determined from numbers of retained and not-retained DCs after magnetic separation. (B) Intracellular iron concentration (pg/cell) of retained DC fractions after magnetic separation. Results in $(A)$ and $(B)$ are mean values \pm SD $\left(n=3\right.$; * $\left.p<0.05 ;{ }^{* *}: p<0.01\right)$. (C) Prussian blue staining of cytospins of magnetically retained and not-retained cells for detection iron deposits. Neutral red dye was used as counterstain. Scale bars, $20 \mu \mathrm{m}$.

Figure 4. Uptake of uncoated and PE-coated ferumoxytol particles into steady state DCs. FL-DCs after uptake of respective MNPs and magnetic separation of MNP-loaded cells were analyzed by flow cytometry. Unlabeled cells were used as control. (A) CD11c+ cells were gated for CD11b and SiglecH as in Figure $2 \mathrm{~B}$ to specify $\mathrm{cDC}$ (black boxes) and pDC (red boxes) subsets. Representative dot plots from 3 independent experiments are shown. (B) Proportion of DC subsets within MNP-labeled FL-DCs compared to unlabeled control FL-DCs. (C) Percentage of MNP-labeled DC subsets within all FL-DCs. Data in (B) and (C) are mean values \pm SEM $\left(n=3 ;{ }^{*} p<0.05,{ }^{* * * *} p<0.0001\right)$. 
Figure 5. Biocompatibility of uncoated and PE-coated ferumoxytol in inflammatory and steady state DCs. Cellular toxicity was analyzed $24 \mathrm{~h}$ after MNP labeling and magnetic sorting using Zombie Aqua staining and analysis by flow cytometry. Bar diagrams show percent of dead cells in (A) GM-DCs, (B) FL$D C$ derived $c D C s$, and $(C)$ FL-DC derived pDCs. Mean values $\pm S D(n=3)$ are plotted $\left({ }^{* *} p<0.01\right)$.

Figure 6. Immunophenotype of inflammatory and steady state DCs upon MNP uptake. MHC II expression as a measure of DC activation was analyzed $24 \mathrm{~h}$ after MNP labeling and magnetic sorting by flow cytometry. Unlabeled cells were used as controls displaying an immature phenotype. LPS stimulation of cells for $24 \mathrm{~h}$ was used as a control for DC activation. Representative data from at least 3 independent experiments are shown. (A) Surface antigen expression of CD11c (gray histograms) and MHC II (open blue histograms) on MNP-labeled GM-DCs. Activation of unlabeled DCs with LPS is depicted in red. Open black histograms show isotype control staining. (B) Bar diagram shows geometric mean of fluorescence intensity (geoMFI) values for MHC II expression \pm SD on GM-DCs. (C-F) MHC II expression on MNP-labeled cDCs and pDCs. (C) DC subsets were identified according to $\mathrm{CD} 11 \mathrm{~b}$ and SiglecH expression and the MHC II expression (open red histograms) determined within specific DC subsets as before (see Fig. 2B). Gray histograms show isotype control staining. Activation of unlabeled cDCs and pDCs by LPS is depicted in blue. (D) MHC II expression on unlabeled and MNP-labeled cDCs (open black histograms) and pDCs (open red histograms). Results of MHC II expression on CDCs (E) and pDCs (F) are shown as geoMFI values \pm SD. 
bioRxiv preprint doi: https://doi.org/10.1101/2021.05.27.445994; this version posted May 27, 2021. The copyright holder for this preprint (which was not certified by peer review) is the author/funder. All rights reserved. No reuse allowed without permission.

A
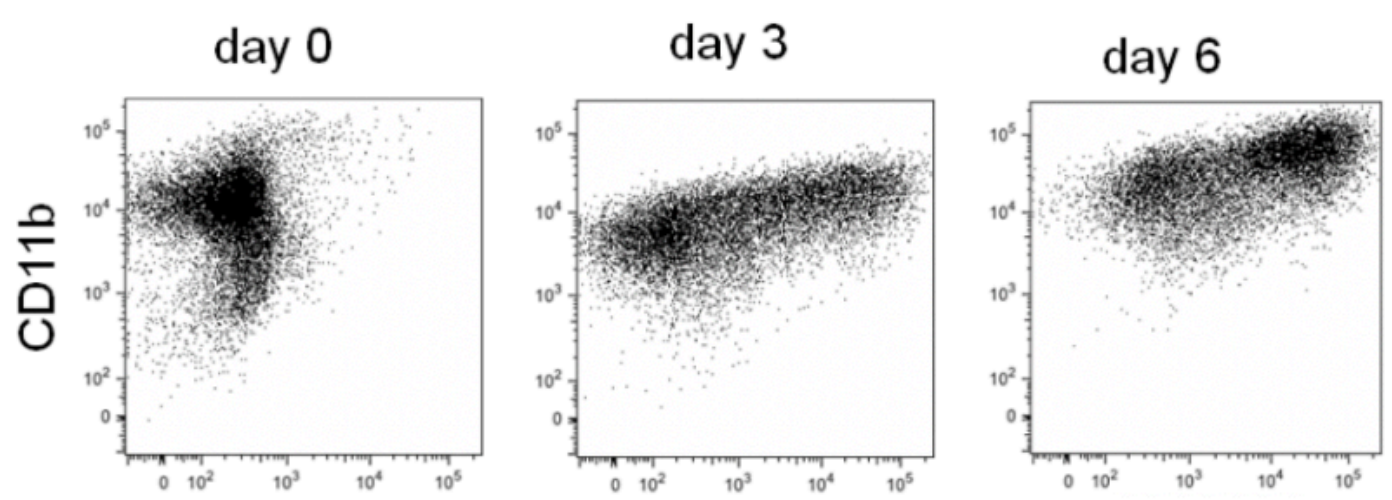

CD11c
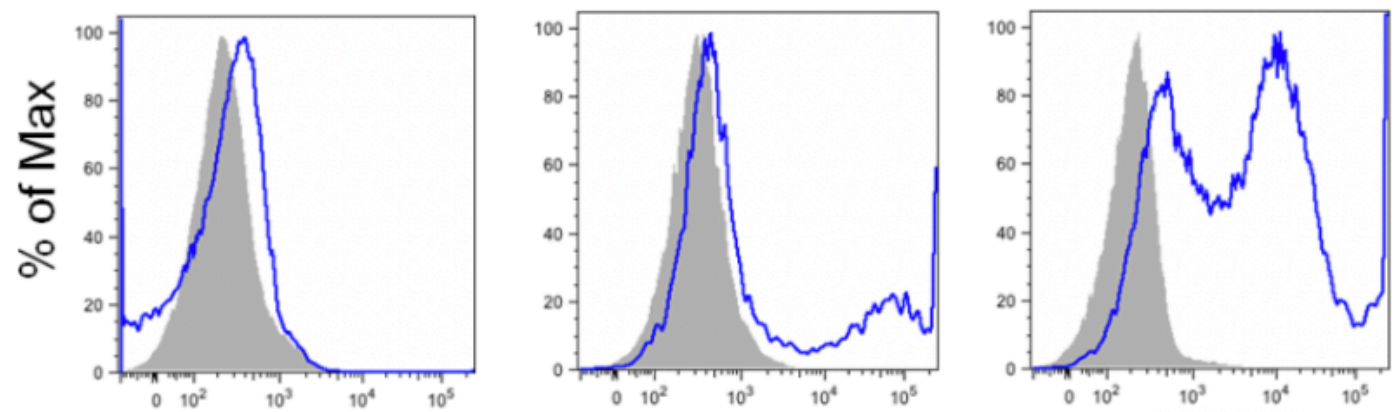

MHC II

B
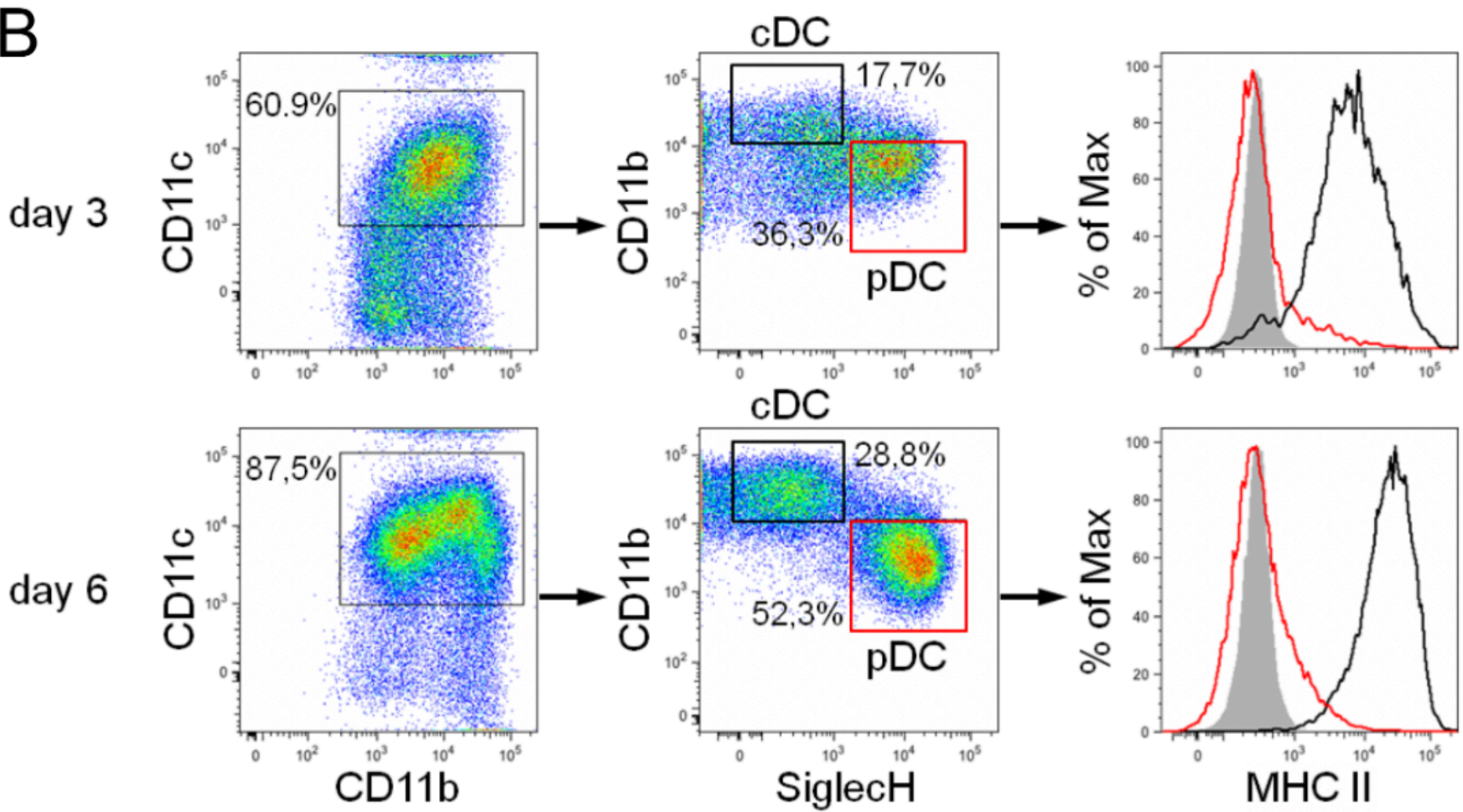

Figure 2 

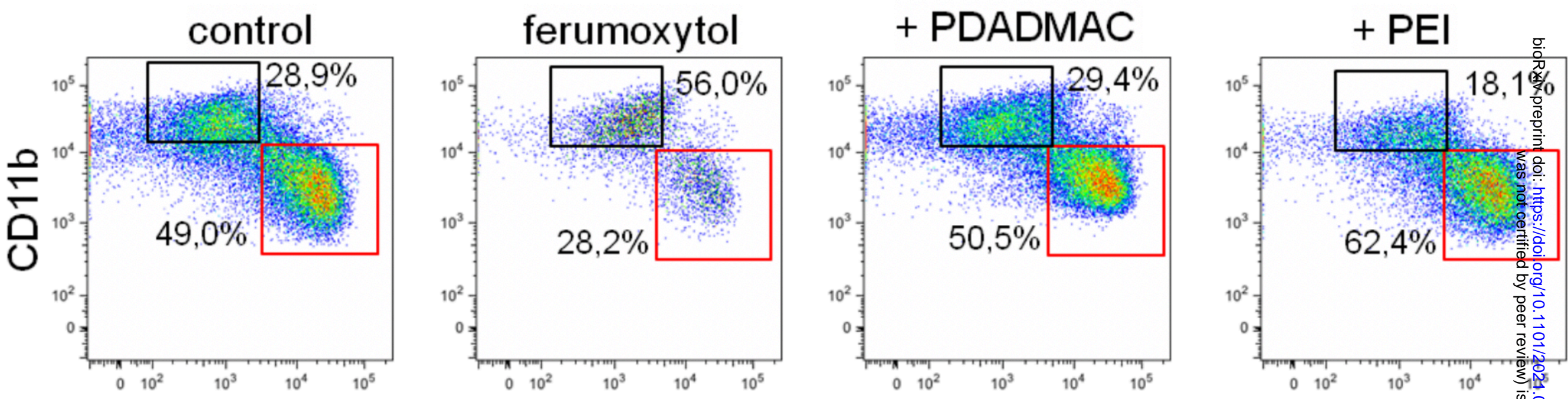

SiglecH
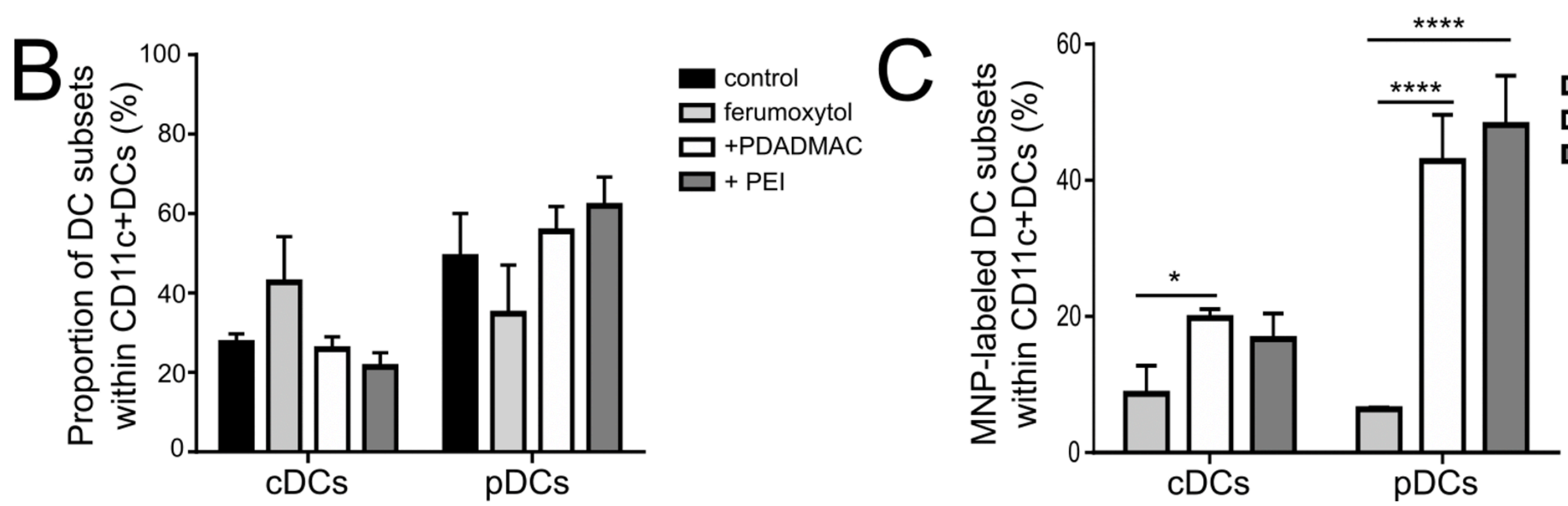

Figure 4 
bioRxiv preprint doi: https://doi.org/10.1101/2021.05.27.445994; this version posted May 27, 2021. The copyright holder for this preprint (which was not certified by peer review) is the author/funder. All rights reserved. No reuse allowed without permission.

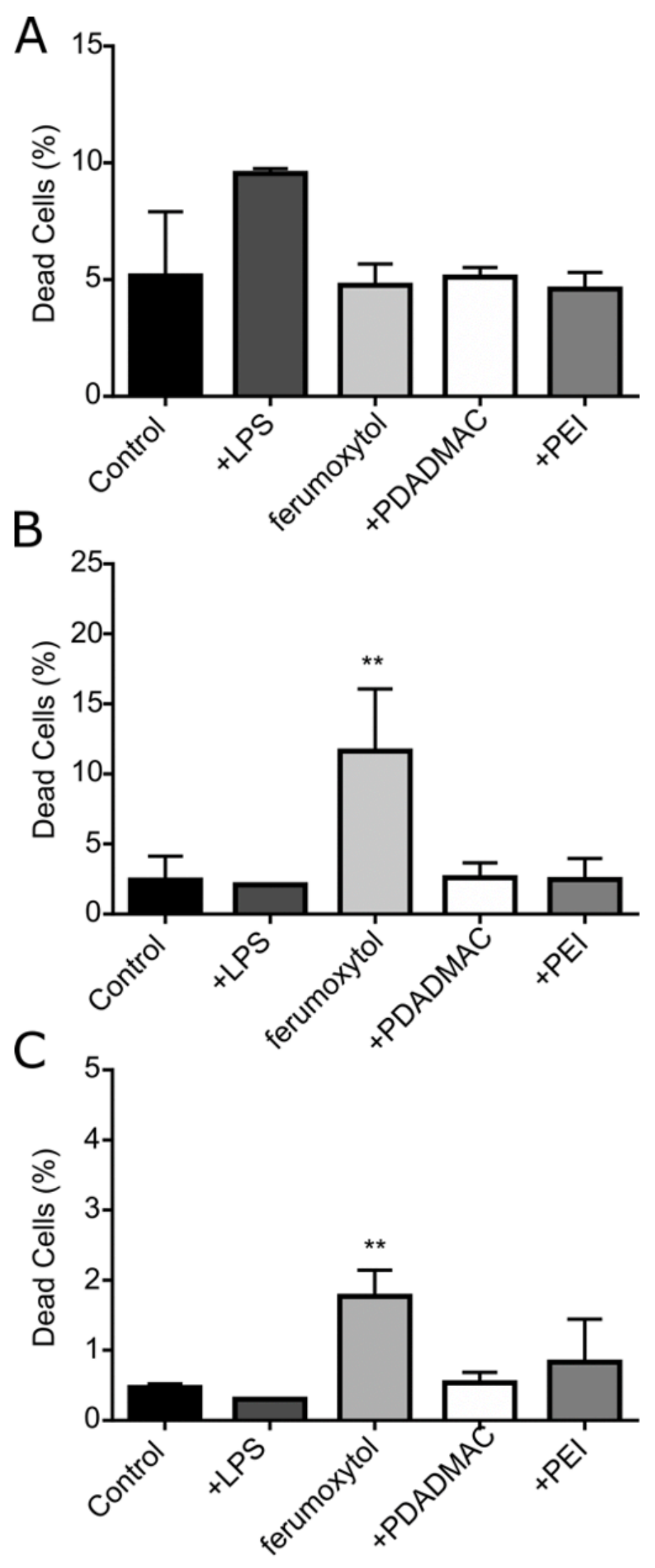

Figure 5 
A

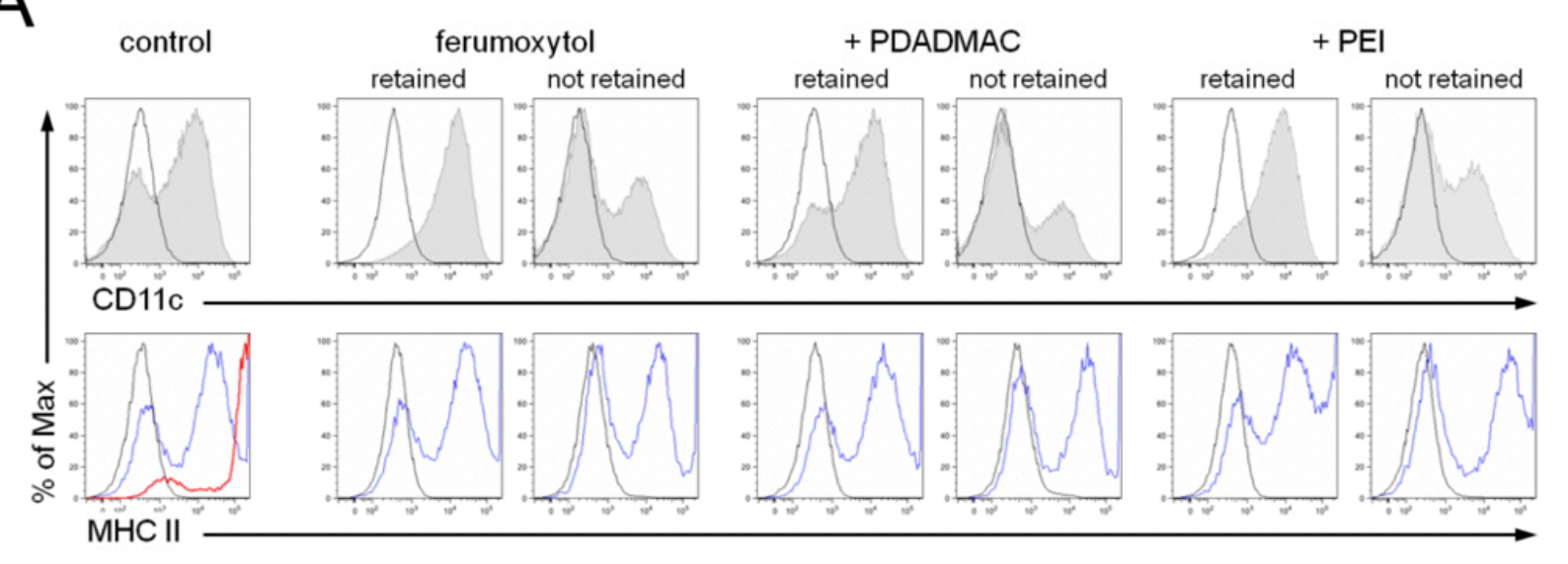

B
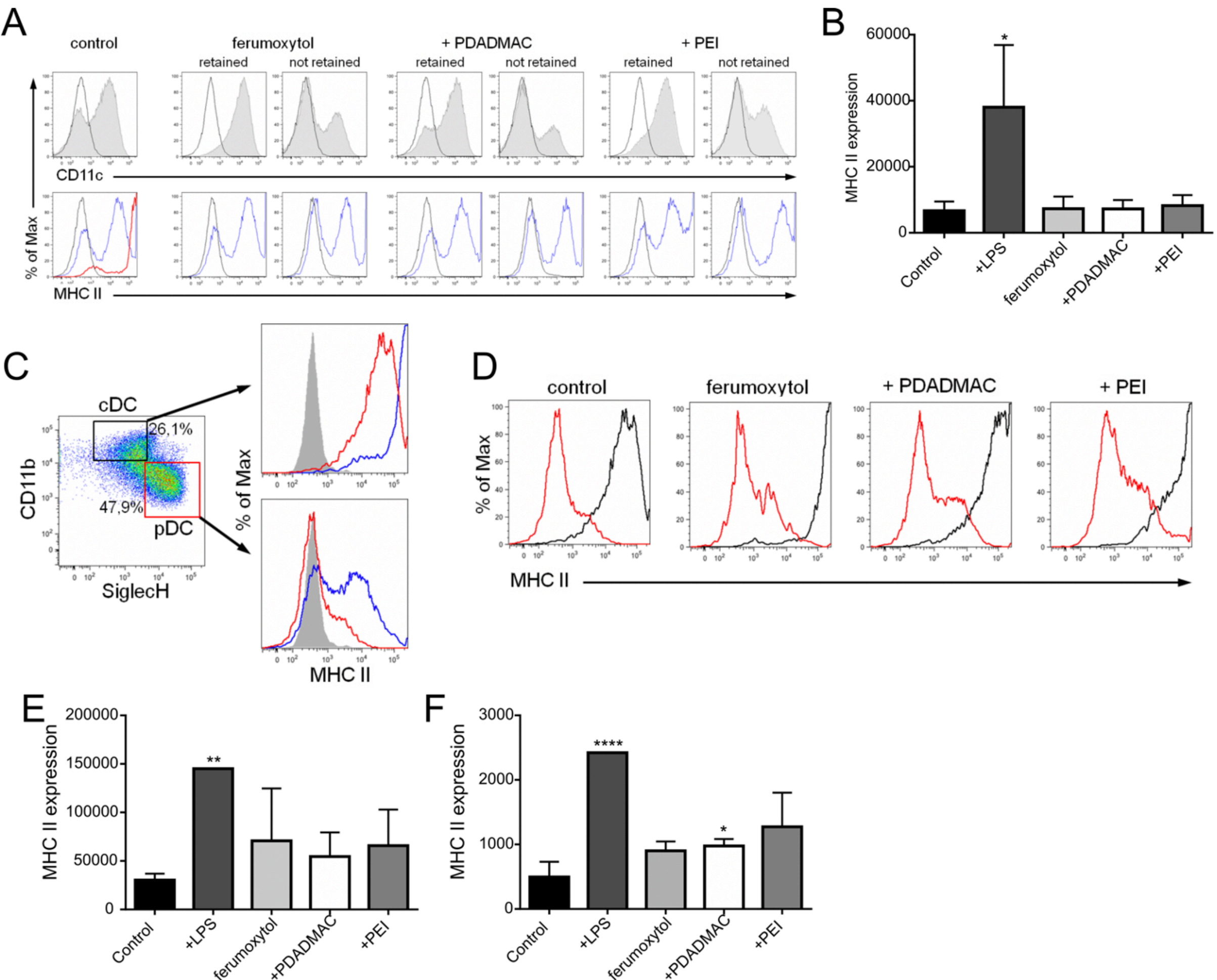

Figure 6 